\title{
On the polarized emission from exciton complexes in GaN quantum dots
}

\author{
S. Amloy, ${ }^{1,2, a)}$ K. F. Karlsson, ${ }^{1}$ T. G. Andersson, ${ }^{3}$ and P. O. Holtz ${ }^{1}$ \\ ${ }_{1}^{1}$ Department of Physics, Chemistry, and Biology (IFM), Linköping University, S-58183 Linköping, Sweden \\ ${ }^{2}$ Department of Physics, Faculty of Science, Thaksin University, Phattalung 93110, Thailand \\ ${ }^{3}$ Applied Semiconductor Physics, Department of Microtechnology and Nanoscience, Chalmers University \\ of Technology, S-41296 Göteborg, Sweden
}

(Received 28 November 2011; accepted 15 December 2011; published online 9 January 2012)

\begin{abstract}
The optical linear polarization properties of exciton complexes in asymmetric Stranski-Krastanov grown $\mathrm{GaN}$ quantum dots have been investigated experimentally and theoretically. It is demonstrated that the polarization angle and the polarization degree can be conveniently employed to associate emission lines in the recorded photoluminescence spectra to a specific dot. The experimental results are in agreement with configuration interaction computations, which predict similar polarization degrees for the exciton and the biexciton (within 10\%) in typical GaN quantum dots. The theory further predicts that the polarization degree can provide information about the charge state of the dot. (C) 2012 American Institute of Physics. [doi:10.1063/1.3675572]
\end{abstract}

A single semiconductor quantum dot (QD) has a potential to generate single photons on demand and individual pairs of entangled photons for quantum cryptography applications. ${ }^{1,2}$ Consequently, it is crucial to be able to investigate the detailed properties of single QDs, and a large number of reports have presented various methods to reduce the number of dots contributing to light emission, e.g., by lowering the dot density trough optimization of the growth temperature, ${ }^{3}$ by controlling the dot nucleation site using micro-structures, ${ }^{4}$ by etching mesa structures containing a random small number of dots, ${ }^{5}$ or by screening the light from unwanted dots by a metal mask on the sample surface. ${ }^{6}$ These methods are typically used in combination with a local probe technique such as micro-photoluminescence ( $\mu \mathrm{PL})$ spectroscopy or cathodoluminescence spectroscopy. For many QD samples, however, the micrometric spatial resolution provided by these techniques is not sufficient to single out an individual QD, and several emission lines that may be attributed to different QDs are typically present in the recorded spectrum. In materials with significant spectral diffusion, groups of emission lines with similar temporal diffusion patterns can be associated with the same QD. ${ }^{7}$ Temporal single photon correlation measurements can also be used to gain knowledge about the spatial origin of the emission lines. ${ }^{8}$

In this work, we investigate the possibility to use the polarization properties of the excitonic emission from $\mathrm{GaN}$ QDs in order to relate the measured emission lines to specific dots. It is found experimentally that both the exciton and the biexciton originating from the same asymmetric QD not only exhibit identical polarization directions, as expected, but also similar degrees of linear polarization. As the available theoretical reports on exciton complexes do not address the polarization properties of asymmetric QDs, ${ }^{9}$ we have also performed configuration interaction computations in order to elucidate the experimental results. Moreover, the theory predicts a dependence of the polarization degree of the trion emission on the charge state. These polarization properties

${ }^{\text {a)} E l e c t r o n i c ~ m a i l: ~ s u p a l u c k @ i f m . l i u . s e . ~}$ can conveniently be used to identify emission lines originating from the same QDs, and the results suggest that precise measurements of the polarization degree yield information about the charge state related to particular emission lines.

A III-V QD normally exhibits some degree of asymmetry, giving rise to anisotropic linear polarization of the optical interband transitions. The polarization of the emitted light is dictated by the symmetry of the QD, ${ }^{10}$ and in the strong confinement regime, the polarization is essentially determined by the dominating single-particle state of the recombining hole. In this case, various exciton complexes with all electrons and holes in the corresponding singleparticle ground states (the neutral exciton, the trions, and the biexciton) would exhibit an identical degree of polarization. However, for typical strained GaN QDs, the strong confinement regime is never reached for the holes. The small splitoff energy and the heavy hole masses in the nitrides result in hole levels spaced merely by about $10 \mathrm{meV}$, i.e., energy separations significantly smaller than the electron-hole and hole-hole direct Coulomb interaction energies $(>50 \mathrm{meV})$. Therefore, in general, Coulomb correlation effects may be dominant, and different degrees of polarization can be expected for, e.g., the neutral single exciton and biexciton confined in the same GaN QDs.

The sample investigated in this work was grown on sapphire [0001] substrate by molecular beam epitaxy (MBE) at a substrate temperature of $720^{\circ} \mathrm{C}$. A $5 \mathrm{~nm}$ AlN nucleation layer was grown on the substrate followed by a $50 \mathrm{~nm} \mathrm{GaN}$ buffer layer and a $50 \mathrm{~nm}$ AlN barrier. The QDs were formed in the Stranski-Krastanov growth mode by 4.5 monolayers deposition of $\mathrm{GaN}$ on this AlN layer. The growth was finalized by an $\mathrm{Al}_{0.5} \mathrm{Ga}_{0.5} \mathrm{~N}$ capping layer.

$\mu \mathrm{PL}$ spectra were measured by using an excitation laser operating at a wavelength of $266 \mathrm{~nm}$. An objective lens focused the laser with high spatial resolution $(\sim 2 \mu \mathrm{m})$ on the sample surface. The sample was placed on a cold-finger inside a cryostat and kept at a temperature of $4 \mathrm{~K}$ by a continuous flow of liquid helium. The photoluminescence emitted from the sample was collected by the same objective lens 
and transferred to a monochromator, which dispersed the signal with a grating of 2400 grooves $/ \mathrm{mm}$. The $\mu \mathrm{PL}$ signal was finally detected by a liquid nitrogen cooled charge-coupled device mounted at the exit port of the monochromator. The spectral resolution of the system was set to $\sim 0.37 \mathrm{meV}$. The polarization analysis system consisted of a rotatable halfwave retardation plate and a fixed linear polarizer, placed in the signal path before the entrance slit of the monochromator.

The $\mu \mathrm{PL}$ spectra of GaN QDs measured for several excitation powers $\left(\mathrm{P}_{\mathrm{ex}}\right)$ are shown in Fig. 1(a). The dominating sharp emission lines at 3590.2, 3610.1, 3616.6, and $3780.9 \mathrm{meV}$ are labeled $\mathrm{A}, \mathrm{XX}, \mathrm{X}$, and $\mathrm{B}$, respectively. The integrated intensities of peaks $\mathrm{A}, \mathrm{B}$, and $\mathrm{X}$ exhibit linear power dependence, whereas the peak XX appears with quadratic power dependence (see Fig. 1(b)). A quadratic dependence is the signature of a biexciton, while a linear dependence is expected for the single exciton. ${ }^{11}$ As the recorded spectra have several emission lines exhibiting single exciton characteristics, it is likely that they originate from different QDs within the laser spot.

In order to associate the biexciton emission $\mathrm{XX}$ to the corresponding single exciton emission A, B, or X, originating from the same $\mathrm{QD}$, additional polarization dependent measurements were employed. The linear polarization was analyzed in the c-plane, and Fig. 2(a) shows the obtained $\mu \mathrm{PL}$ spectra for three different angles $(\theta)$ of the polarization analyzer (as indicated in the figure). Corresponding polar plots of the integrated intensity (I) as a function of $\theta$ are shown in Figs. 2(b) and 2(c) for peaks A, XX, X, and B. The polarization angle $(\varphi)$ of the emission was determined by fitting the measured data with the formula $\mathrm{I}(\theta)$ $=I_{\min } \sin ^{2}(\theta-\varphi)+I_{\max } \cos ^{2}(\theta-\varphi)$, where $I_{\min }, I_{\max }$, and $\varphi$ are fitting parameters $\left(I_{\max }>I_{\min }\right)$. Peaks $X$ and XX exhibit the same polarization angle $\varphi=30^{\circ}$, corresponding to maximal intensity, while the angles for peaks $\mathrm{A}$ and $\mathrm{B}$ are $\varphi=10^{\circ}$ and $\varphi=150^{\circ}$, respectively. There is no polarized fine-structure splitting resolved for any of the investigated peaks. ${ }^{6}$ The degree of linear polarization $\mathrm{P}=\left(\mathrm{I}_{\max }-\mathrm{I}_{\min }\right) /$ $\left(I_{\max }+I_{\min }\right)$ for peaks $\mathrm{X}, \mathrm{XX}, \mathrm{A}$, and $\mathrm{B}$, as extracted from the fits, are $91 \%, 86 \%, 90 \%$, and $98 \%$, respectively. ${ }^{12}$ The (a)

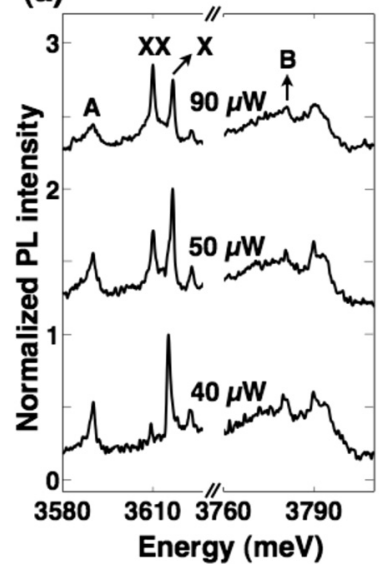

(b)

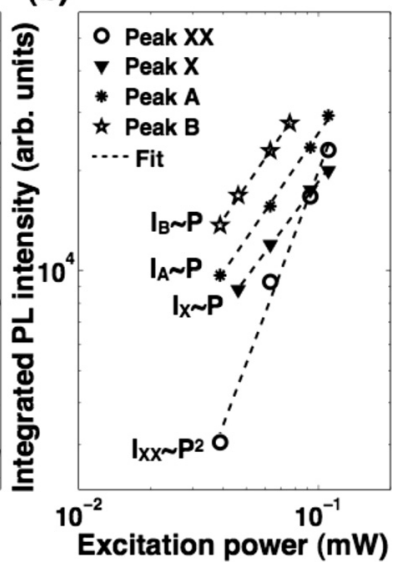

FIG. 1. (a) $\mu \mathrm{PL}$ spectra of GaN QDs measured with different excitation power, as indicated in the figure. (b) The integrated peak intensity of the emission lines $\mathrm{XX}, \mathrm{X}, \mathrm{A}$, and $\mathrm{B}$ versus the excitation power. (a)

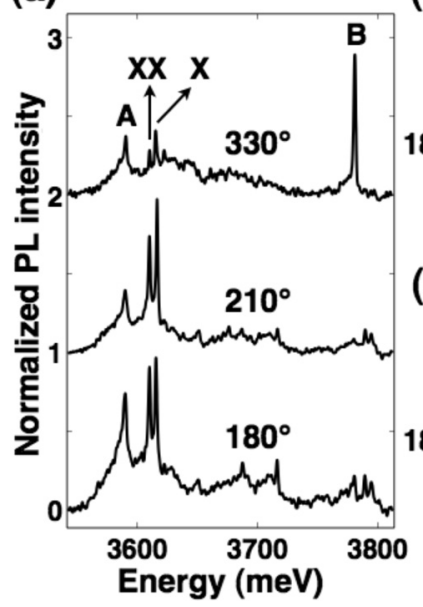

(b)

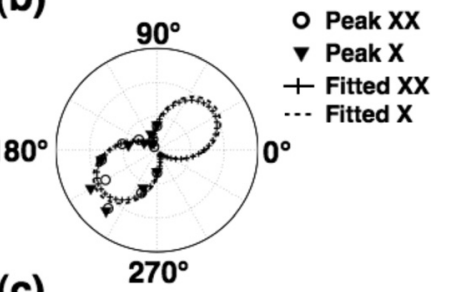

(c)

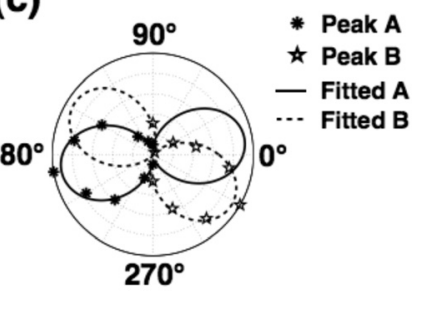

FIG. 2. (a) Polarization resolved $\mu \mathrm{PL}$ spectra of GaN QDs recorded for different angles $180^{\circ}, 210^{\circ}$, and $330^{\circ}$ of the linear polarization analyzer. (b), (c) Polar plots of the integrated peak intensities of the emission lines XX, X, $\mathrm{A}$, and $\mathrm{B}$ as a function of the polarization analyzer angle along with curve fittings.

high degree of linear polarization obtained for all emission lines indicates that all the measured QDs are strongly asymmetric. The apparently random polarization angles $\varphi$ obtained for the emission lines indicate that the asymmetry of the QDs does not exhibit any clear preferential direction. The fact that $\mathrm{X}$ and $\mathrm{XX}$ exhibit the same polarization angle suggests that they originate from the same QD. Thus, the power dependence combined with the polarization results allow the conclusion that $\mathrm{X}$ and $\mathrm{XX}$ correspond to the emission of a single exciton and a biexciton from the same QD, with a biexciton binding energy of $6.5 \mathrm{meV}$. Consequently, the emission lines A and B are attributed to excitonic emissions from two different QDs.

Interestingly, both $\mathrm{X}$ and $\mathrm{XX}$ exhibit very similar degrees of linear polarization $\left(P_{X}=91 \%\right.$ and $\left.P_{X X}=86 \%\right)$, consistent for a $\mathrm{QD}$ in the strong confinement regime. However, as mentioned above, typical nitride based QDs have closely spaced hole levels preventing them from being in a strong confinement regime. Thus, substantial Coulomb induced mixings of the hole states are expected, which, in general, can alter the degree of polarization with different strength for different exciton complexes.

Numerical computations were employed in order to elucidate the effects of Coulomb interactions on the degree of linear polarization of exciton complexes in typical $\mathrm{GaN}$ QDs. The single particle states of wurtzite GaN/AlN QDs were computed with a 8 band k.p Hamiltonian ${ }^{13,14}$ and discretized by finite differences. The Hamiltonian includes strain effects, computed with continuum elastic theory, as well as the internal electric fields originating from the spontaneous and piezoelectric polarization. ${ }^{14}$ The resulting single-particle states were used to determine the interband dipole matrix elements and the electron-hole, hole-hole, and electron-electron Coulomb scattering matrix elements, for subsequent computation of the quantum states and optical emission spectra of the exciton complexes by means of configuration interactions. ${ }^{15}$ Coulomb correlation effects are intrinsically included in the configuration interactions, which 
were expressed in a basis formed from 8 electron states and 16 hole states (including spin). The material parameters were taken from Ref. 16, using a valence band offset of $0.85 \mathrm{eV}$ between GaN and AlN and, for simplicity, a constant and isotropic dielectric constant of 9.8. The QD of height $h$ was assumed be lens-like with an elliptical base area, standing on a wetting layer of thickness $t$. The lateral extension of the QD is determined by the mean radius $r$, enclosing the area $\pi r^{2}$, and a QD symmetry parameter $\alpha$ defined as the ratio between the minor and major axes of the elliptic base, as shown in the top inset of Fig. $3(\alpha=1$ implies a lens-shaped QD with circular base of radius $r$ ).

Optically active GaN QDs must be thin in order to provide sufficient electron-hole overlap despite the presence of a strong built-in electric field. The typical dot height is about $1 \mathrm{~nm}$ (Ref. 5) and such thin QDs have a small aspect ratio, near $0.1{ }^{3}$ Computations were performed for two QD models, one relatively large dot with $r=6 \mathrm{~nm}(h=t=1 \mathrm{~nm})$ and one smaller dot with $r=4 \mathrm{~nm}(h=t=0.75 \mathrm{~nm})$, with predicted exciton emission energies at 3290 and $3775 \mathrm{meV}$, respectively. Fig. 3 shows the computed degree of linear polarization of the exciton $(\mathrm{X})$, the biexciton $(\mathrm{XX})$, and the trions $\left(\mathrm{X}^{ \pm}\right)$, for the large $\mathrm{QD}$, with the symmetry parameter $\alpha$ ranging from 0.2 to 1 . All exciton complexes exhibit the expected progressive increase of the polarization degree when $\alpha$ is reduced, and the polarizations of all the complexes follow each other closely. In particular, the difference in polarization degree between $\mathrm{X}$ and $\mathrm{XX}$ always remains well below $10 \%$, as shown by the open circles in the lower inset of Fig. 3. This result is fairly insensitive to the exact QD size, as shown by the shaded circles in the lower inset of Fig. 3, as computed for the smaller dot. The largest discrepancy in polarization degree among the studied complexes is found between the positive and the negative trions, exhibiting a difference up to $16 \%$ for the small QDs with moderate asymmetry. This suggests that accurate measurements of the polarization degree yield information about the charge state

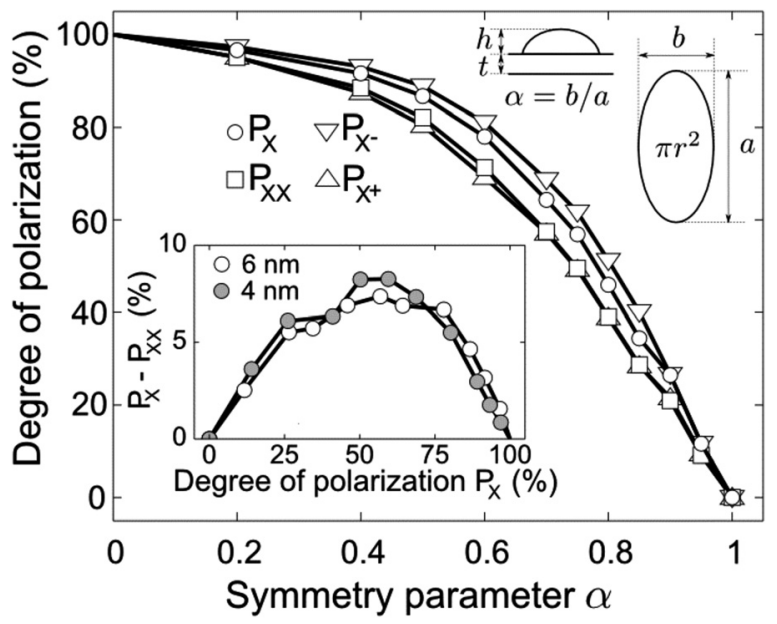

FIG. 3. Computed degree of linear polarization for the exciton $\mathrm{P}_{\mathrm{X}}$ (circles), biexciton $\mathrm{P}_{\mathrm{XX}}$ (squares), positive trion $\mathrm{P}_{\mathrm{X}+}$ (up-pointing triangles), and negative trion $\mathrm{P}_{\mathrm{X}-}$ (down-pointing triangles) as a function of the symmetry parameter $\alpha$, keeping $r=6 \mathrm{~nm}$. The top inset shows a schematic illustration of the QD model, and the lower inset shows the computed difference in degree of linear polarization between the exciton and the biexciton for two QD sizes ( $r=6 \mathrm{~nm}$ and $r=4 \mathrm{~nm}$ ), plotted versus the polarization degree of the exciton emission. Solid lines serve as guide to the eye. associated with the emission lines. Note that although trions are well-known for III-arsenide based dots, ${ }^{17}$ they have not yet been experimentally identified in the spectra of IIInitride QDs.

The experimental data shown in Fig. 2 exhibit a high degree of linear polarization $(\sim 90 \%)$ for both excitons and biexcitons. Such a high polarization degree is consistent with the computations for strongly asymmetric QDs $(\alpha \approx 0.45)$. Moreover, for such asymmetric QDs very similar degrees of linear polarization for excitons and biexcitons are theoretically predicted (within $4 \%$ for $P_{X} \geq 90 \%$, see Fig. 3), in full agreement with the polarization degrees measured for $\mathrm{X}$ and XX (see Fig. 2). Thus, it is demonstrated that the various Coulomb interactions, which occur within an excitonic complex in a GaN QD, do not significantly alter the polarization degree. This opens the possibility to exploit also the polarization degree, in addition to the polarization direction, in order to identify the excitonic emissions originating from a specific QD.

In summary, it has been demonstrated that the polarization of the light emitted by asymmetric Stranski-Krastanov QDs can provide additional spatial information beyond the micrometric resolution of standard $\mu \mathrm{PL}$. The $\mathrm{X}$ and $\mathrm{XX}$ emissions of individual GaN QDs could thereby be distinguished in spectra recorded for an ensemble of dots. Moreover, computations of the linear polarization confirm that the degree of polarization is expected to be very similar for $\mathrm{X}$ and $\mathrm{XX}$ in typical GaN QDs. In particular, for strongly asymmetric dots exhibiting a high exciton polarization degree $\left(P_{X} \geq 90 \%\right)$, the polarization degree of the biexciton $\left(\mathrm{P}_{\mathrm{XX}}\right)$ is merely discrepant by a few percent with the single exciton $\left(\mathrm{P}_{\mathrm{X}}-\mathrm{P}_{\mathrm{XX}} \leq 4 \%\right)$, in accordance with the presented experimental data. The largest discrepancy is predicted between the positive and negative trions, which may be used to identify trions and their charge states in the polarization resolved spectra of GaN QDs.

This work has been supported by a Ph.D. scholarship from Thaksin University in Thailand for S. Amloy, grants from the Swedish Research Council (VR), the Swedish Foundation for Strategic Research (SSF) funded Nano-N consortium and the Knut and Alice Wallenberg Foundation. The authors are thankful to R. Farivar for assistance in growth, K. H. Yu for assistance in PL measurement, and M. A. Dupertuis for enlightening discussions.

${ }^{1}$ C. Kurtsiefer, P. Zarda, M. Halder, H. Weinfurter, P. M. Gorman, P. R. Tapster, and J. G. Rarity, Nature (London) 419, 450 (2002).

${ }^{2}$ F. Ding, R. Singh, J. D. Plumhof, T. Zander, V. Křápek, Y. H. Chen, M. Benyoucef, V. Zwiller, K. Dörr, G. Bester, A. Rastelli, and O. G. Schmidt, Phys. Rev. Lett. 104, 067405 (2010).

${ }^{3}$ C. Adelmann, B. Daudin, R. A. Oliver, G. A. D. Briggs, and R. E. Rudd, Phys. Rev. B 70, 125427 (2004).

${ }^{4}$ C. W. Hsu, A. Lundskog, K. F. Karlsson, U. Forsberg, E. Janzén, and P. O. Holtz, Nano Lett. 11, 2415 (2011).

${ }^{5}$ D. Simeonov, A. Dussaigne, R. Butté, and N. Grandjean, Phys. Rev. B 77, 075306 (2008).

${ }^{6}$ S. Amloy, Y. T. Chen, K. F. Karlsson, K. H. Chen, H. C. Hsu, C. L. Hsiao, L. C. Chen, and P. O. Holtz, Phys. Rev. B 83. 201307(R) (2011).

${ }^{7}$ V. Türck, S. Rodt, O. Stier, R. Heitz, R. Engelhardt, U. W. Pohl, D. Bimberg, and R. Steingrüber, Phys. Rev. B 61, 9944 (2000).

${ }^{8}$ A. Malko, M. H. Baier, E. Pelucchi, D. Y. Oberli, K. Leifer, D. Chek-alkar, and E. Kapon, Appl. Phys. Lett. 85, 5715 (2004).

${ }^{9}$ S. Tomić and N. Vukmirović, Phys. Rev. B 79, 245330 (2009). 
${ }^{10}$ R. Bardoux, T. Guillet, B. Gil, P. Lefebvre, T. Bretagnon, T. Taliercio, S. Rousset, and F. Semond, Phys. Rev. B 77, 235315 (2008).

${ }^{11}$ K. Brunner, G. Abstreiter, G. Böhm, G. Tränkle, and G. Weimann, Phys. Rev. Lett. 73, 1138 (1994).

${ }^{12}$ It cannot be excluded that these values are underestimated by up to $\sim 2 \%$ due to polarization cross-talk, possibly caused by imperfections in the optics, light scattering and birefringence of the sample.

${ }^{13}$ S. L. Chuang and C. S. Chang, Phys. Rev. B 54, 2491 (1996).
${ }^{14}$ M. Winkelnkemper, A. Schliwa, and D. Bimberg, Phys. Rev. B 74, 155322 (2006).

${ }^{15}$ A. Barenco and M. A. Dupertuis, Phys. Rev. B 52, 2766 (1995).

${ }^{16}$ I. Vurgaftman, J. R. Meyer, and L. R. Ram-Mohan, J. Appl. Phys. 89, 5815 (2001).

${ }^{17}$ E. S. Moskalenko, K. F. Karlsson, P. O. Holtz, B. Monemar, W. V. Schoenfeld, J. M. Garcia, and P. M. Petroff, J. Appl. Phys. 92, 6787 (2002). 